\title{
Physics of Instabilities in Radiatively Driven Stellar Winds
}

\section{S.P. Owocki, Bartol Research Institute/ University of Delaware \\ J.I. Castor, Lawrence Livermore National Laboratory \\ G.B. Rybicki, Harvard-Smithsonian Center for Astrophysics}

One type of variability that is ubiquitous among luminous blue stars is that associated with their massive stellar winds. Ultraviolet spectral lines show variable narrow absorption components that may arise from dense shells or clumps in the wind; the observed soft Xray and non-thermal radio emission from these stars may be produced in embedded wind shocks associated with these shells. The line-driving of such winds is known to be strongly unstable, and recent numerical simulations of the dynamical, nonlinear evolution of such instabilities indicate that such shocks and dense shells do indeed form (Owocki, Castor, and Rybicki 1988; hereafter OCR). However, the structure computed in these dynamical simulations is quite different, and in some sense opposite, what was anticipated in earlier heuristic shock models (e.g. Lucy 1984, Krolik and Raymond 1985, Abbott 1988); the highest speed material is very rarefied, not dense, and the strongest shocks that form are of the reverse, not forward, type. In subsequent simulations we have found that the detailed character of this structure can depend on many variables, but this tendency always to form high speed rarefied waves and reverse shocks is quite robust. It is thus important that we have a clear understanding of the reasons for this tendency, and it is on this point that we focus our comments here.

As pointed out in OCR, this formation of high speed rarefactions is a natural consequence of the fact that the inward-propagating (relative to the fluid) radiative-acoustic mode, for which the density and velocity variations are nearly opposite in phase, is much more unstable than the outward mode, for which density and velocity are nearly in phase. In a formal, mathematical sense, this last result was already clear from the linear stability analysis of Owocki and Rybicki (1984), but we now offer the following brief explanation of its physical basis:

For ordinary acoustic waves, the velocity and density perturbations are exactly in phase for the outward mode, but opposite in phase for the inward mode. Addition of a perturbed line force with a component in phase with the velocity causes work to be done on the wave, thereby tending to amplify it. However, during this amplification the velocity and density can no longer remain exactly in or exactly out of phase. This is because mass continuity requires that the associated intrinsic temporal decrease or increase in the perturbed density must be balanced respectively by a positive or negative divergence (i.e. 
gradient) in the perturbed velocity. The sense of the required phase shift is such that the perturbed pressure force (which varies as the negative of the perturbed density gradient) now has a component that opposes the line-force for the outward mode, but reinforces it for the inward mode. The result is that the outward mode is only weakly unstable, whereas the inward mode is strongly unstable. Amplification of such unstable inward mode waves naturally leads to wind structure in which the highest velocity material is very rarefied, and kinematic steepening of this structure naturally leads to the formation of the reverse shocks.

Note that the only nonlinear effect here is the simple kinematic steepening of the large amplitude waves into shocks. Relatively complicated, dynamical, nonlinear effects like line shadowing, which played such a prominent role in earlier heuristic shock models, play a rather minor role here, only affecting various details of the structures. Thus this very robust property of the nonlinear wind structure is really a straightforward consequence of the properties of the linear instability. If we had only been clever enough, we could have predicted it! Of course, we usually aren't so clever, and it is the great value of numerical simulations that, when carefully analyzed, they can teach us basic physics that we could have known without ever doing them.

We thank J. Cassinelli for thoughtful questions that helped stimulate us to develop a sounder physical understanding of our numerical results. The computations described here were performed at the San Diego Supercomputer Center and the Lawrence Livermore National Laboratory. This work was performed in part under the auspices of the U.S. Department of Energy by the Lawrence Livermore National Laboratory under Contract No. W-7405-ENG-48. S.P.O. acknowledges partial support from NSF grant AST 8611824.

\section{References}

Abbott, D.C. 1988, in Solar Wind VI, V.J. Pizzo, ed., NASA Conference Proceeding, in press.

Krolik, J. and Raymond, J.C. 1985, Ap. J., 298, 660.

Lucy, L.B. 1982, Ap. J., 255, 286.

Owocki, S. P. and Rybicki, G. B. 1984, Ap. J., 284, 337.

Owocki, S. P., Castor, J. I., and Rybicki, G. B. 1988, Ap. J., 335, in press. (OCR). 